\title{
INTERVENCIÓN MÉDICO-PSICOLÓGICA CON PACIENTE Y FAMILIARES EN UN CASO DE CÁNCER GINECÓLOGICO EN CUIDADOS PALIATIVOS ONCOLÓGICOS
}

\author{
I HAVE INTENTION TO GET A MAMMOGRAM: STAGES OF ADOPTION FOR \\ MONITORING MAMMOGRAPHY IN WOMEN OF DIFFERENT SOCIAL AND \\ CULTURAL BACKGROUND
}

Ma Patricia Acinas, Milton Olallo Arango y Santiago Antón

Hospital San Juan de Dios. Burgos.

Resumen

Objetivos: El objetivo del presente artículo es mostrar el trabajo que se hizo desde el punto de vista médico y psicológico en un caso de carcinoma seroso papilar primario extraovárico. Se buscó el control de síntomas físicos y psicológicos, así como potenciar las habilidades y recursos de paciente y familiares.

Problemas psicológicos: La paciente mostraba un proceso de negación adaptativa y limitaciones psicofuncionales (astenia, disminución de autonomía personal, fístula, dolor) que afectaban a su calidad de vida, además de ansiedad, bajo estado de ánimo y rumiaciones cognitivas. Todo esto tenía una repercusión en la familia en miedos, ansiedad, incertidumbre.

Método de intervención: Las actuaciones se llevaron a cabo considerando al paciente y a la familia como unidad de intervención. Se realizó trabajo en equipo y coordinación diaria entre los profesionales desde el mismo momento del ingreso de la paciente hasta el fallecimiento. Además se llevaron a cabo seguimientos posteriores con la familia en relación a la elaboración del duelo. Se empleó Counselling e intervenciones psicológicas desde el modelo cognitivo conductual.

Resultados: Se consiguió minimizar reacciones de ansiedad, cognitivas y afectivas, valorar recursos existentes en toda la unidad fa-
Abstract

Objetives: To show the efforts made from medical and psychological point of view in a case of extraovarian serous papillary carcinoma. We looked for the control of physical and psychological symptoms, as well as the increase of skills and resources in the patient and her family.

Psychological problems: The patient showed a process of adaptative denial and some psycho-functional limitations (asthenia, decrease of autonomy, fistula, and pain) which affect her quality of life, in addition of anxiety, low state of mood and repeated thoughts. All of them had a repercussion in her family such as some fears, anxiety and incertitude.

Method of intervention: The actions carried out considered patient and her family like a unit of intervention. Teamwork was done, as well as everyday coordination among the professionals from the moment of admission the patient in the hospital to the death. Also some follow-up with relatives were borne with regard to grief working- out. It was applied Counselling and psychological interventions from a cognitive-behavioral model.

Results: We achieved to minimize reactions of anxiety, cognitives and affectives, to appreciate the existent resources in the whole family unit, decrease of worries, improve the

\section{Correspondencia:}


miliar, disminución de preocupaciones, mejorar la comunicación paciente y familia, adaptación progresiva a la cercanía de un desenlace.

Palabras clave: Cáncer ginecológico, cuidados paliativos, intervención médico psicológica, trabajo en equipo, Atención familiar. communication between patient and her family, progressive adaptation to the proximity of denouement.

Keywords: Gynecological cancer, palliative care, medical psychological intervention, teamwork, family attention.
No permitas que lo que no puedes hacer interfiera en lo que sí puedes hacer.

(J. Wooden)

\section{INTRODUCCIÓN}

Cuando aparece una enfermedad en la familia se produce un gran impacto a varios niveles: personal, familiar, laboral, cultural, espiritual. Atender las necesidades de las personas en cualquier momento vital es una responsabilidad moral, pero cuando se hace en la fase final de la vida constituye un reto importante para la adaptación humana.

El carcinoma seroso papilar primario peritoneal extraovárico es un raro tumor epitelial maligno que se desarrolla en el peritoneo que recubre el abdomen y la pelvis y se caracteriza por carcinomatosis abdominal ${ }^{(1,2)}$. Es similar al carcinoma ovárico seroso con respecto a la presentación clínica, apariencia histológica, patrón de extensión, tratamiento y pronostico Se ha informado de esta entidad con diversos nombres incluyendo el carcinoma papilar de la superficie serosa del peritoneo, el adenocarcinoma de Müller extraovárico ${ }^{(3,4)}$. Fue descrita por primera vez por Swerdlow en 1959 como «el mesotelioma del peritoneo pélvico ${ }^{(5)}$. Esta patología es responsable de entre el $7 \%$ y $15 \%$ de los casos de cáncer de ovario ${ }^{(3)}$.

En Cuidados Paliativos, los problemas de comunicación son percibidos por el personal sanitario como los más importantes en el trato con los pacientes ${ }^{(6)}$ y con sus respectivas familias, porque no es fácil transmitir malas noticias y al mismo tiempo la esperanza de que se pueden llevar a cabo actuaciones en pro del paciente. Además nos encontramos con una desventaja fundamental porque el tiempo asistencial puede ser muy corto, por lo que la eficacia de cada intervención es crucial y los planes terapéuticos a largo plazo no suelen ser realizables ${ }^{(7)}$.

\section{INTEGRACIÓN DEL PSICÓlOGO EN EL EQUIPO MÉDICO}

En el Hospital San Juan de Dios de Burgos la figura del psicólogo esta integrado dentro del equipo facultativo como un miembro más del mismo ${ }^{(8)}$, con tareas y funciones propias diferenciadas, de apoyo y en coordinación, con los integrantes del equipo asistencial. Esto corresponde a lo que algunos autores Ilaman Modelo Fundacional de integración del psicólogo o psicooncólogo ${ }^{(9)}$ dentro de los servicios de oncología, y por extensión dentro de las unidades de cuidados paliativos, como parte del equipo interdisciplinar buscando una atención integral y la operatividad del funcionamiento.

La psicóloga comparte espacios, salas de trabajo y reuniones en las que participan los médicos, sus observaciones e intervenciones psicológicas se incorporan a la Historia Clínica informatizada en el mismo apartado que los médicos del hospital. También lleva un BuscaTeléfono, de igual manera que el resto de los facultativos, lo cual permite su rápida localización, por ejemplo ante emergencias psicológicas o acompañamiento en duelo de manera precoz (inmediatamente después del éxitus). Psicología posee protocolos de actuación ${ }^{(10)}$ independientes en los que se indican las fun- 
ciones que lleva a cabo: Acogida de paciente y familiares, Acompañamiento al ingreso, Alta hospitalaria, Apoyo a Médicos, Consultas, Interconsultas, Intervención Personal del Hospital, Intervención en Duelo, un Urgencias psicológicas, Acompañamiento a visita médica, Apoyo a Personal Sanitario.

Los dos aspectos más novedosos en nuestro hospital son la incorporación del psicólogo en la Visita Médica, con la participación activa del mismo en la entrevista al paciente; y la realización de ingreso-acogida-apoyo en la anamnesis del mismo a la llegada al hospital, así como el acompañamiento al médico tratante en la transmisión a la familia de la situación del paciente a la llegada al centro hospitalario y el manejo de aspectos psicológicos iniciales desde el momento del ingreso en el hospital.

\section{DESCRIPCIÓN DEL CASO CLÍNICO}

Se trata de una paciente femenina de 71 años de edad, casada, vive con su esposo, madre de tres hijos, 4 nietos. Reside entre Burgos capital y un pueblo de la Provincia de Burgos. Procede del servicio de Oncología del hospital general correspondiente el día 29-07-2010 en juicio clínico de:

- Carcinoma seroso papilar primario extraovárico con progresión peritoneal estadio III. moral.

- Fistula Recto-Vesical por Infiltración Tu-

- Enfermedad Neoplásica Terminal.

Se diagnostica posteriormente, durante su ingreso en nuestro hospital una fístula recto vesical a pared abdominal por progresión tumoral.

Los tratamientos previos que recibió para el tratamiento de su enfermedad oncológica (antes del ingreso en nuestro hospital) fueron:

- Intervención quirúrgica: histerectomía, doble anexectomia, linfoadenectomia y extirpación de epiplón mayor y peritoneo.

- Quimioterapia: cinco líneas de tratamiento citostático. Por el deterioro funcional presentado y la progresión tumoral, el servicio de oncología del hospital de referencia decide no continuar con ningún tratamiento activo oncológico.

La paciente es alérgica al cloramfenicol, sulfamidas, beta lactámicos. Heparinas de bajo peso molecular, cítricos y todo lo que contenga anisakis.

A su llegada al hospital la paciente se encuentra consciente, orientada, colaboradora; muy asténica, deambulaba con apoyo de otra persona.

Objetivos Médicos al Ingreso en el Hospital San Juan de Dios

- Mantener a la paciente confortable y con control de síntomas, tanto como sea posible y lo necesite ${ }^{(11)}$.

- Control de síntomas médicos frecuentes en esta paciente: emesis, infecciones, dolor, estreñimiento, disnea, molestias gástricas ${ }^{(12)}$.

- Colaboración, comunicación e información a la familia.

Las Escalas Funcionales que se usan ampliamente en Oncología y en Cuidados Paliativos para indicar el estado clínico en que se encuentra el paciente son las siguientes:

- Karnosky ${ }^{(13)}$ : Desde 40\% (necesidad de cuidados y asistencia) hasta $20 \%$ (muy enferma, necesidad de tratamiento paliativo activo).

- ECOG ${ }^{(14)}$; Desde 2 (paciente encamado menos del 50\% del tiempo y satisfacción de necesidades personales solo) hasta 4 (encamado el $100 \%$ del tiempo, ayuda para todas las actividades básicas de vida diaria, ABVD).

- RANKIN(15): Desde 3 (Incapacidad moderada) hasta 5 (Incapacidad severa, dependiente).

En la paciente a la que nos referimos en este artículo, la evolución en las tres escalas se expone en la tabla 1.

\section{Control del Dolor y analgesia}

Uno de los aspectos fundamentales en la paciente, era poner los medios farmacológicos y no farmacológicos para limitar la aparición de dolor. Para ello se emplearon varios medicamentos con intención analgésica

En la tabla 2, se muestran de manera pormenorizada cómo se fue variando el tipo de 
Tabla 1. Valoración escalas funcionales en relación a la progresión de la enfermedad.

\begin{tabular}{|c|c|c|c|}
\hline PERIODO & KARNOSKY & ECOG & RANKIN \\
\hline INGRESO Y PRIMEROS DÍAS & $40 \%$ & 2 & 3 \\
\hline APARICIÓN 2 ${ }^{a}$ FÍSTULA & $30 \%$ & 3 & 4 \\
\hline ÚLTIMOS MOMENTOS $^{\mid}$ & $20 \%$ & 4 & 5 \\
\hline
\end{tabular}

\section{Tabla 2. Requerimientos de analgesia de la paciente según los diversos momentos temporales.}

\begin{tabular}{|c|c|c|c|}
\hline MOMENTO TEMPORAL & $1^{\text {ER ESCALÓN }}$ & $2^{\circ}$ ESCALÓN & $3^{\text {ER ESCALÓN }}$ \\
\hline INGRESO Y PRIMEROS DÍAS & Paracetamol 3gr/día & Nada & $\begin{array}{l}\text { MST 200mg/día } \\
\text { Sevredol } 60 \mathrm{mg} / \text { cada } 4 \mathrm{~h} \\
\text { (Rescate) }\end{array}$ \\
\hline 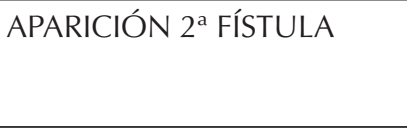 & Paracetamol 3gr/día & Nada & $\begin{array}{l}\text { MST } 260 \mathrm{mg} / \mathrm{día} \\
\begin{array}{l}\text { Sevredol } \\
\text { (Rescate) }\end{array}\end{array}$ \\
\hline ÚLTIMOS MOMENTOS & Paracetamol 3gr/día & Nada & $\begin{array}{l}\text { CLM } 100 \text { mg /día (IV) } \\
\text { CLM } 80 \text { mg/día (rescate) } \\
\text { (IV) }\end{array}$ \\
\hline
\end{tabular}

medicación analgésica administrada, así como las dosis de la misma, en función de los síntomas referidos por la paciente y/o la familia, u observados por el equipo asistencial (incluyendo médico, enfermeras, auxiliares, psicóloga). Se usaron fundamentalmente fármacos de primer y tercer nivel en la Escalera Analgésica de la OMS. Se incrementó fundamentalmente la medicación opiácea, tanto la medicación pautada periódicamente como la dosis correspondiente a los rescates (medicación extra solicitada entre dosis de medicación pautada periódicamente).

\section{Control de síntomas: otros medicamentos empleados}

Aunque el control del dolor se convierte en objetivo prioritario en esta paciente, es necesario implementar medicación para controlar otros síntomas que aparecen o para prevenir la aparición de otros síntomas que pueden provocar disconfort en la paciente. Los fármacos que se emplearon y su función terapéutica se enumeran a continuación.

- Buscapina. Antiespasmódico.

- Dacortín®. Fortecortín ${ }^{\circledR}$ Corticoesteroides.

- Primperán ${ }^{\circledR}$. Antiemético

- Omeprazol. Protector gástrico.

- Aldactone®. Diurético

- Atrovent. Salbutamol. Inhaladores aerosoles broncodilatadores.

- Mycostatin $®$, Oraldine $®$. Mucositis y molestias orales

- Micralax $®$ (microenema). Salvacolina. Para estreñimiento.

- Oxígeno medicinal.

\section{Psicofármacos}

En lo que se refiere al control de síntomas psicológicos y para favorecer el bienestar psíquico, se emplearon los siguientes psicofármacos. Se describe también su nombre comercial, su acción y grupo terapéutico.

- Deprax®; Trazodona. ASIR (Antagonista 
$5 \mathrm{HT}_{2}$ e inhibición de Recaptación 5HT)

- Lexatín $\mathbb{~}$; Bromazepam. Benzodiacepina acción media

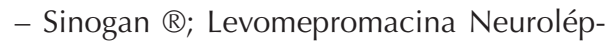
tico fenotiacínico

En esta paciente, aumenta progresivamente el deterioro funcional y disminución de los índices de Karnofsky y ECOG (ver tabla 1), con la imposibilidad de deambular, necesidad de mayor control de síntomas, infecciones recurrentes respiratorias y renales, deterioro de la función respiratoria, hasta su fallecimiento a los 58 días de su ingreso, el 24-09-2010.

\section{Evolución y progresión de la enfermedad de base}

El ingreso en el hospital, en la Unidad de Cuidados Paliativos, implica el cuidado de manera integral biopsicosocial por parte de todo el equipo. Para que este tipo de atenciones se puedan Ilevar a cabo, muchas veces es necesario conocer y tener en cuenta acontecimientos anteriores, que permitan entender y manejar de manera personalizada las manifestaciones en los tres niveles (biológico, psicológico y social). Por este motivo en la figura 1 se han ordenado de manera cronológica los acontecimientos vitales de la paciente desde el momento de su nacimiento hasta que se produce el fallecimiento. Se han establecido dos líneas, en la superior los eventos previos al ingreso en nuestro hospital, y en la línea inferior los eventos que tuvieron lugar mientras estuvo hospitalizada en el Hospital San Juan de Dios.

\section{ASPECTOS PSICOLÓGICOS DE LA PACIENTE}

Después de concretar en los apartados anteriores los aspectos que pertenecen más al ámbito médico, queremos profundizar en este apartado en los aspectos psicológicos que se

\section{Figura 1. Línea de la vida de la paciente con acontecimietos biográficos y hospitalarios.}

\section{Línea de la vida}

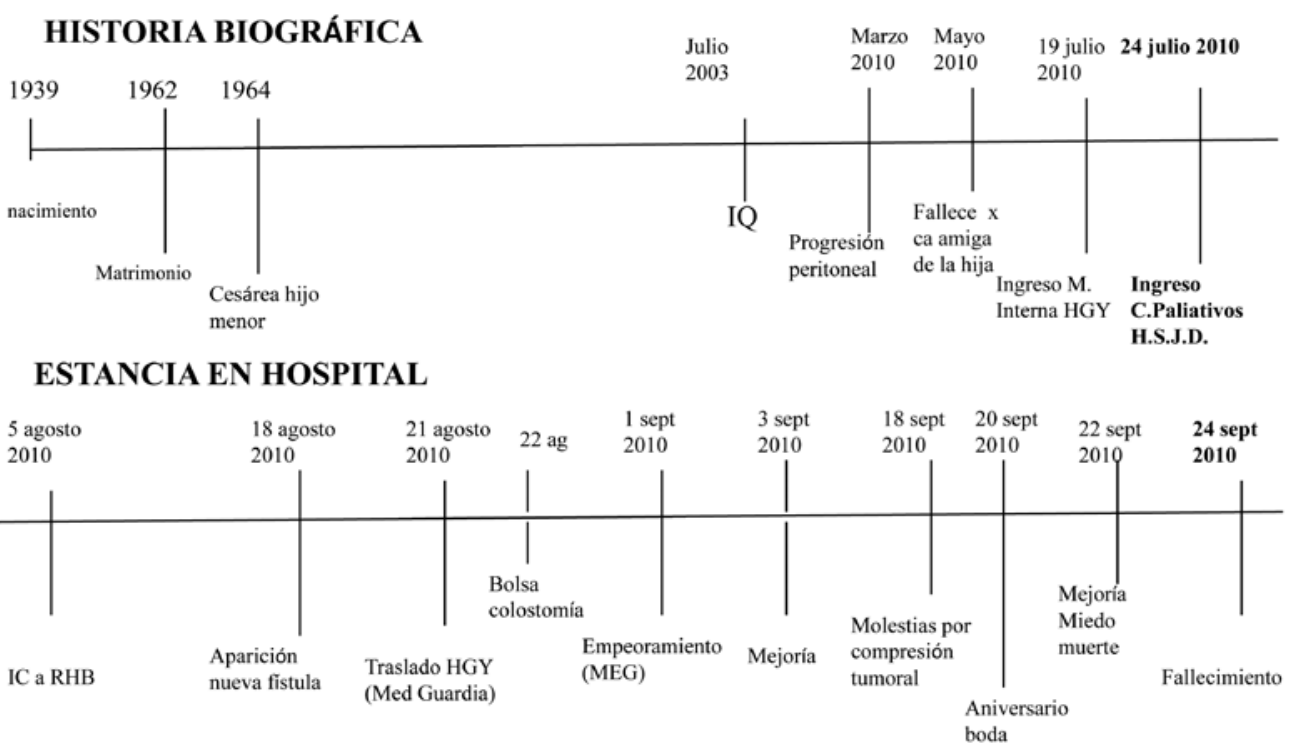


observaron en la paciente desde el inicio, y como se fueron produciendo cambios en la misma, por la progresión de la enfermedad y por las intervenciones médico-psicológicas, y en general de todo el equipo asistencial.

Cuando se realiza la exploración psicológica de la paciente ${ }^{(16)}$, el mismo día del ingreso y los días posteriores se muestran los siguientes rasgos psicológicos:

Orientación en las tres esferas, colaboradora en las actividades que se le proponían y los temas de evaluación psicológica (aunque no eran percibidos por ella de esa manera, porque se creaba un clima distendido que favorecía la colaboración).

- Pensamiento: rumiaciones cognitivas, tendencia negativista, distorsiones cognitivas, anticipación, focalización selectiva, catastrofismo.

- Lenguaje: adecuado, coherente, fluido (no surgieron alteraciones de tipo sensoperceptivo en la evaluación inicial)

- Emociones y sentimientos: ansiedad, tristeza, pena, impotencia. Miedos. Labilidad emocional. Tristeza

- Afrontamiento: preocupación ansiosa, espíritu de lucha, negación adaptativa. Planificación de objetivos a largo plazo no realistas. Necesidad y búsqueda de contacto físico afectivo (asociado a baja tolerancia a soledad). Ligeros rasgos obsesivos (observables en las rutinas, aspectos decisionales -tendencia a buscar decisiones seguras ya conocidas anteriormente-), como elemento de autoseguridad.

Debido a las características de la paciente, con elevada astenia, y ansiedad de evaluación, no se aplicaron pruebas psicológicas estandarizadas que hubieran bloqueado la relación terapéutica. Se empleó observación participante y no participante, y entrevista semiestructurada (tanto con el paciente como con su familia) para valorar aspectos psicológicos personales y de la interacción. Para ello se realizaron visitas cortas casi diarias a la paciente durante la primera semana.

A medida que continuaba la estancia en el hospital se iban haciendo reevaluaciones periódicas de su situación psicofuncional para adaptar las estrategias y ajustar los objetivos terapéuticos. En ocasiones se mantenían y en otras ocasiones se cambiaba y se pasaba a otra fase. Por cuestiones de claridad expositiva se ha dejado para más adelante la exposición de los tres momentos de intervención diferenciados (junto a las técnicas empleadas en cada uno de ellos), porque corresponden a aspectos médicos y psicológicos diferenciados.

La paciente percibía su enfermedad como amenaza y tenía miedo a la soledad ${ }^{(17)}$ y lo mostraba mediante aspectos fundamentalmente no verbales. Se estableció un buen vínculo terapéutico desde inicio, tanto con el médico tratante como con la psicóloga del hospital. La misma paciente hacía frecuentemente las peticiones de acompañamiento psicológico de manera directa, a través de su familia y cuidadora, o mediante el personal de enfermería y auxiliares de planta.

Durante el periodo de evaluación se realizaron visitas conjuntas de médico y psicóloga. Posteriormente a las mismas se intercambiaban impresiones por parte de ambos profesionales para favorecer el manejo conjunto, y adecuar las intervenciones de cada profesional, teniendo en cuenta la opinión clínica del otro, por ejemplo el médico tenía en cuenta la opinión de la psicóloga para adecuar las dosis de benzodiacepinas, y la psicóloga conocía en qué momentos se incrementaban algunos medicamentos para que la intervención psicológica sirviera de coadyuvante a la actuación médica interviniendo en los momentos más idóneos o reforzando información facilitada por el médico en su presencia.

El diagnóstico psicopatológico según los criterios del DSM-IV-TR ${ }^{(18)}$ es el siguiente:

- Eje I: F 43.22 Trastorno adaptativo mixto ansioso-depresivo. Crónico ( $>6$ meses)

- Eje II: Sin diagnóstico.

- Eje III: Neoplasias. Carcinoma seroso extraovárico con progresión peritoneal

- Eje IV: Problemas relativos al grupo primario de apoyo (perturbación familiar)

- Eje IV: EEAG= 70 (síntomas leves), Agosto $2010 \mathrm{EEAG}=60$ (síntomas moderados), Sep- 
Figura 2. Genograma familiar.

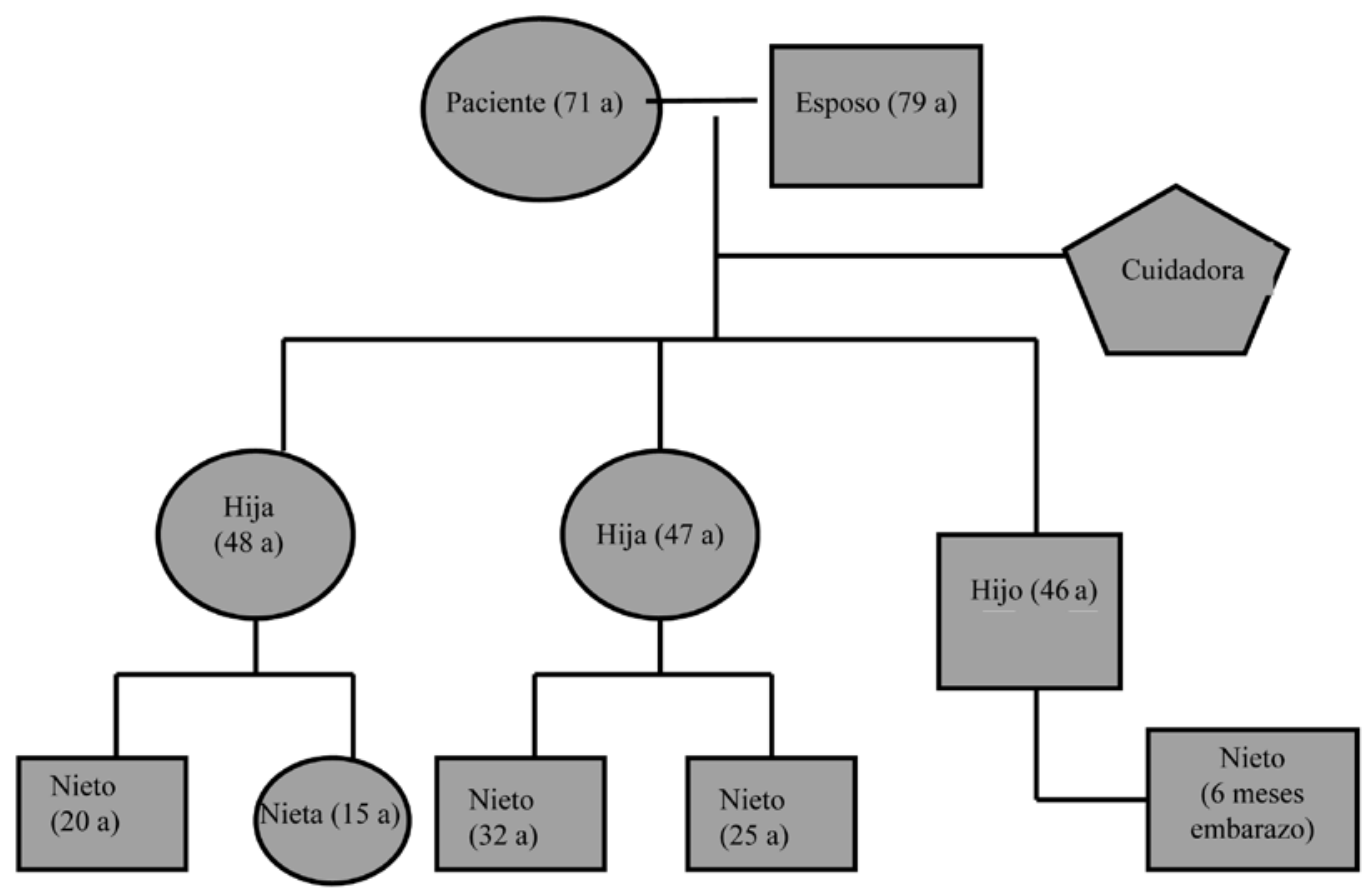

tiembre 2010; EEAG $=80$ (esperables y transitorios)

Tras la descripción de los aspectos familiares relacionados con el caso de la paciente se plasmará de manera comparativa cuales fueron los objetivos terapéuticos, médicos y psicológicos, tanto con paciente como con familia. Hacerlo de esta manera nos permite tener una visión de conjunto de todo el proceso y de cómo fueron variando las actuaciones sanitarias de los profesionales implicados desde el inicio y en función de los aspectos que fueron apareciendo a nivel médico, como la segunda fístula, que marcó una serie de cambios a diversos niveles que se concretarán más adelante.

\section{ASPECTOS PSICOLÓGICOS DE LA FAMILIA}

En el caso de esta paciente las relaciones familiares entrañaban cierta complejidad, tanto por el modelo familiar, como por las interac- ciones presentes en ese momento, como por las características psicológicas de los diversos miembros. Expondremos los rasgos más importantes y los que influyeron en la toma de decisiones terapéuticas en relación al manejo médico y psicológico, técnicas empleadas, forma y manera en que se facilitaba información médica de la paciente a los familiares, actuaciones psicológicas individuales con algún miembro de la familia. El acompañamiento familiar al final de la vida ${ }^{(6)}$ se torna en ocasiones dificultoso por la disparidad y singularidad de personalidades de los miembros de la familia y por las diversas situaciones que pueden ir surgiendo $y$ que muchas veces, no son del todo previsibles.

En las entrevistas iniciales con la familia se aprecian los siguientes aspectos que definen al grupo familiar:

- Estructura familiar (ver figura 2): se aprecian algunas alianzas entre algunos miembros de la familia, derivadas de conflictos antiguos no resueltos, en que unos miembros de la fa- 


\section{Figura 3. Análisis funcional y modelo de formulación del caso.}

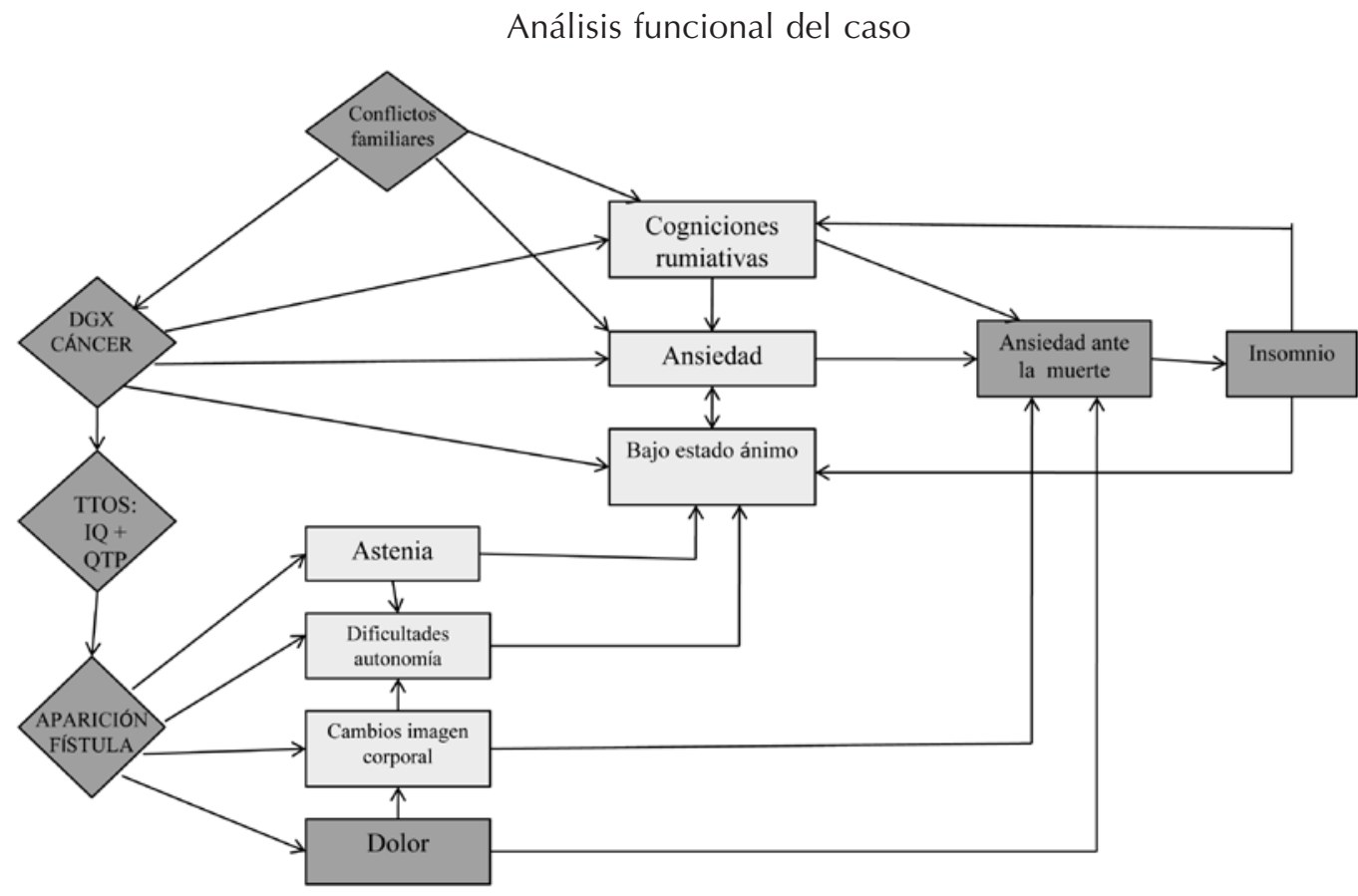

milia tomaron parte por una persona y otros miembros por otra persona. El resultado de esos conflictos y cómo se resolvieron entonces influyó en las manifestaciones emocionales de paciente y familiares, y las atribuciones casuales que se realizaban por parte de todos (paciente y familia) sobre algunas conductas de los demás.

- Marido (cumplieron 46 años matrimonio), manifestaba una baja expresión emocional inicial, bloqueo en la expresión cognitiva y afectiva.

- Dos hijas que muestran ansiedad, implicación en los cuidados y apoyo mutuo entre hermanas ante la enfermedad de la madre.

- Un hijo, bastante reacio a intervención psicológica, que percibe con desconfianza. Se mantienen pocas entrevistas con él.

- Una cuidadora (mantiene una relación cercana con la familia). Implicada, cariñosa y un buen apoyo para la paciente

- Nuera (esposa del único hijo) percibida por la paciente con ambivalencia, (desconcier- to ante pérdida de su apoyo durante la enfermedad y hospitalización, cuando anteriormente la relación era muy fluida). Estaba embarazada de pocos meses. No fue a visitarla al hospital a la paciente.

Para entender mejor cómo interaccionan las diversas variables psicológicas en la paciente, y analizar las influencias y contingencias en el caso, se estableció un modelo de análisis funcional psicológico, que se expone en la figura 2. Sirvió como modelo de actuación especializado y personalizado, para analizar los factores que mantenían ciertas conductas y decidir sobre qué elementos era prioritaria la intervención del equipo.

En gris claro y con figura en forma de rombo aparecen los factores asociados con la enfermedad (el diagnostico, los tratamientos y la aparición de la fístula) y no asociados (los conflictos familiares), que no se pueden modificar y que ejercen una influencia en la paciente. Estos factores interactúan entre sí y producen 
Tabla 3. Profesionales que participaron en la coordinación del caso.

\begin{tabular}{|c|c|}
\hline INTRAHOSPITALARIA & EXTRAHOSPITALARIA \\
\hline$>$ Médico tratante & $>$ Psicóloga AECC. \\
$>$ Psicóloga & $>$ Psicóloga Equipo Valoración de la \\
$>$ Supervisoras Enfermería & $>$ \\
$>$ Informes Servicio de Oncología \\
$>$ Auxiliares & \\
$>$ Trabajadora Social & \\
$>$ Servicio de Rehabilitación. & \\
\hline
\end{tabular}

otras consecuencias a nivel psicológico. En rectángulo con gris oscuro aparecen los dos síntomas que aparecieron y tuvieron tratamiento médico y psicológico (ansiedad e insomnio). Y en rectángulo de gris más oscuro aparece la ansiedad ante la muerte como factor central que recibe influencias de todos los factores anteriores de manera directa o indirecta, y que fue el objeto principal de tratamiento desde todas las vertientes.

Hay un apartado de manifestaciones más físicas (astenia, dificultades de autonomía, cambios en la imagen corporal y dolor) y otro apartado de aspectos más psicológicos (cogniciones rumiativas, ansiedad y bajo estado de ánimo). Se producen abundantes interacciones entre ambos tipos de factores, que llegan a producir influencias e interdependencias en todos los aspectos anteriores.

Aunque la intervención y coordinación fundamental, dada la complejidad del caso, se realizó entre médico y psicóloga, hubo otros profesionales que aportaron su quehacer especializado en este caso (ver tabla 3). Muchos de ellos personal del hospital (enfermeras, auxiliares, trabajadora social), pero también profesionales externos al mismo, a los que hubo que consultar (servicio de oncología del hospital general de referencia) informar (Psicóloga del equipo de valoración de discapacidad de la Junta de Castilla y León) o con los que fue necesario coordinación porque se estaba tratando a parte de la unidad familiar (como la psicóloga de la AECC que veía en consulta periódicamente al marido de la paciente). Se efectuó la labor de coordinación sin interferir en los objetivos de cada psicóloga y tampoco se solaparon intervenciones. Se respetó la intimidad de los usuarios y el intercambio de información se hizo teniendo en cuenta todos los criterios de ética y deontología profesional, así como la protección de datos.

\section{INTERVENCIÓN INTERDISCIPLINAR CON PACIENTE Y FAMILIA}

Los objetivos globales de la intervención conjunta, a lo largo de todo el proceso, fueron los siguientes:

- Proporcionar el cuidado médico y psicológico como parte de un todo integrado.

- Intentar satisfacer las necesidades físicas, psicológicas, sociales y espirituales de la paciente y su familia.

En esta primera parte del epígrafe de intervención se va a hablar de los diversos tipos de intervención, porque algunos tipos de ellas son poco frecuentes dentro del ámbito de los cuidados paliativos y la Psicooncología. La organización del hospital y la cercanía-buena comunicación entre servicio médico y de psicología permite que algunos tipos de intervenciones (como las conjuntas) se pueden llevar a cabo frecuentemente.

Las actuaciones que se llevaron a cabo en el caso pueden considerarse de varios tipos: 


\section{Intervenciones con la paciente:}

- Individuales. De tipo médico o psicológico. Ambas intervenciones podían hacerse a la paciente sola o en presencia de un familiar o de la cuidadora. Se llevaban a cabo en diversos horarios (momentos de comidas, paseo; tanto por la mañana como por la tarde)

- Conjuntas de médico y psicóloga, con la paciente al mismo tiempo, cada uno con su rol profesional, de una manera ordenada, respetando los turnos de palabra y los objetivos terapéuticos de sendos profesionales.

- Sucesivas; intervención médica en primer lugar y psicológica después, o viceversa, psicológica y luego médica, siempre en el mismo día. Asociado a comunicación y coordinación entre los dos profesionales en el intervalo entre ambas entrevistas o al final de las mismas.

\section{Intervenciones con la familia de la paciente}

- Individuales. De tipo médico o psicológico. Con uno o varios miembros de la misma familia a la vez.

- Conjuntas de médico y psicóloga, con uno o varios familiares (transmisión de información de ambos tipos, comunicación de micromalas noticias, asociado a apoyo psicológico, ventilación emocional con ambos profesionales), en ocasiones a petición familiar, otras razones.

- Sucesivas; intervención médica en primer lugar y psicológica después, o viceversa, psicológica y luego médica, siempre en el mismo día. Asociado a comunicación y coordinación entre los dos profesionales entre ambas entrevistas o al final de las mismas. Muchas veces finalizando con una reunión familiar conjunta con ambos profesionales.

Se llevaron a cabo conversaciones frecuentes con la familia sobre el proceso oncológico para luego poder hablar con la paciente sobre los aspectos de la enfermedad, evolución y síntomas; esto permite comprender las actitudes de la familia y paciente ante la misma ${ }^{(19)}$ y po- der corregir o ajustar la situación a la realidad, o explicar aspectos que ellos pueden percibir como confusos. Estas actuaciones con la familia se llevaban a cabo en función de su disposición y receptividad, mediante previa cita, o de manera proactiva por parte de la familia o de los miembros del equipo. En algunas ocasiones, por ejemplo, era necesario transmitir malas noticias o micromalas noticias y se favorecía que tanto psicóloga como médico estuvieran presentes en la intervención familiar con los familiares o allegados que deseaban recibir información sobre la evolución de la paciente. En otras ocasiones era la misma familia la que solicitaba hablar sobre sus preocupaciones y la presencia de ambos profesionales conjugaba la información médica específica con la contención emocional necesaria para la familia angustiada por el pronóstico infausto. En Cuidados Paliativos las intervenciones son menos programadas, más cercanas a una intervención en crisis, al manejo emocional y a la prevención del desbordamiento emocional. Es difícil planificar algunas intervenciones tanto con paciente como con familia, porque en el día a día pueden surgir situaciones imprevistas que obliguen a replantear los planes y acciones terapéuticas previstas.

En la tabla 4 se recoge el trabajo específico realizado por parte de la psicóloga, y se expone de manera organizada cuáles fueron los objetivos terapéuticos y de qué manera (tareas psicológicas) se diseñó el cumplimiento de los mismos. Se han establecido tres momentos temporales diferenciados que requirieron diferenciación en el enfoque psicológico y también médico: los primeros días de la hospitalización (que conlleva un periodo de adaptación a las normas y rutinas hospitalarias), la aparición de la segunda fístula (la que comunicaba la anterior a piel, con lo que implicaba para la paciente ver como de su abdomen salían mezclados orina, heces y otras secreciones; curas más dolorosas) y un tercer momento, los últimos días, en que se produjo el empeoramiento que condujo a la muerte, con las implicaciones para la paciente pero sobre todo para la familia. 


\section{Tabla 4. Objetivos generales y tareas psicológicas en los distintos momentos temporales del caso.}

\begin{tabular}{|c|c|c|}
\hline $\begin{array}{l}\text { MOMENTO } \\
\text { TEMPORAL }\end{array}$ & OBJETIVOS PSICOLÓGICOS & $\begin{array}{l}\text { METODOLOGÍA DE INTERVENCIÓN } \\
\text { PSICOLÓGICA }\end{array}$ \\
\hline \multirow[t]{2}{*}{$\begin{array}{l}\text { INGRESO Y } \\
\text { PRIMEROS } \\
\text { DÍAS DE LA } \\
\text { HOSPITALIZACIÓN }\end{array}$} & $\begin{array}{l}\text { Paciente: Sentar bases para la } \\
\text { confianza y rapport terapéutico. }\end{array}$ & $\begin{array}{l}\text { Coordinación diaria con médico } \\
\text { tratante }^{(11)} \text {. } \\
\text { Acompañamiento a Visita Médica. }\end{array}$ \\
\hline & 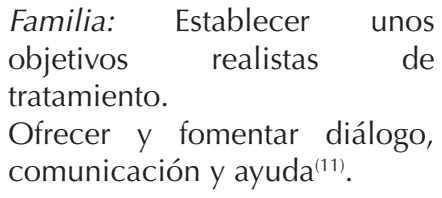 & $\begin{array}{l}\text { Mantenimiento de entrevistas con } \\
\text { miembros de la familia y cuidadora. } \\
\text { Información conjunta a la familia con } \\
\text { médico tratante }\end{array}$ \\
\hline \multirow[t]{2}{*}{$\begin{array}{l}\text { APARICIÓN } 2^{\mathrm{a}} \\
\text { FÍSTULA }\end{array}$} & $\begin{array}{l}\text { Paciente: Potenciar las } \\
\text { habilidades de afrontamiento } \\
\text { de la paciente. }\end{array}$ & $\begin{array}{l}\text { Coordinación diaria con médico } \\
\text { tratante }{ }^{(11)} \text {. } \\
\text { Realización de Intervenciones conjuntas } \\
\text { o sucesivas con el médico tratante. } \\
\text { Apoyo psicológico durante las curas de } \\
\text { la fístula (por parte de DUEs) }\end{array}$ \\
\hline & $\begin{array}{l}\text { Familia: Fomentar lo positivo y } \\
\text { dar seguridad en la interacción } \\
\text { con la paciente. }\end{array}$ & $\begin{array}{l}\text { Realización de entrevistas de } \\
\text { seguimiento con familia y cuidadora. } \\
\text { Información a la familia con médico } \\
\text { tratante sobre situación de la paciente. }\end{array}$ \\
\hline \multirow[t]{2}{*}{$\begin{array}{l}\text { ÚLTIMOS } \\
\text { MOMENTOS }\end{array}$} & 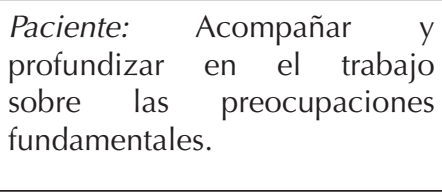 & $\begin{array}{l}\text { Coordinación diaria con médico } \\
\text { tratante }^{(11)} \text {. } \\
\text { Intervenciones conjuntas o sucesivas } \\
\text { con el médico tratante. }\end{array}$ \\
\hline & $\begin{array}{l}\text { Familia: Apoyar a la familia para } \\
\text { mejorar la relación y disminuir } \\
\text { la sobrecarga por cuidado } \\
\text { hospitalario prolongado }{ }^{(11)}\end{array}$ & $\begin{array}{l}\text { Intervenciones psicológicas cortas y } \\
\text { frecuentes con los familiares directos y } \\
\text { cuidadora. } \\
\text { Información con médico tratante } \\
\text { informando a la familia sobre situación } \\
\text { de la paciente. }\end{array}$ \\
\hline
\end{tabular}

En cada uno de los tres apartados anteriormente mencionados se especifica cuáles fueron los objetivos y tareas en relación al paciente y en relación con los familiares de la misma. Las tareas se entienden como la metodología de trabajo que se empleó para conseguir los objetivos anteriores, En la siguiente tabla se explica- rá de manera más minuciosa cuáles fueron las técnicas y estrategias empleadas desde el punto de vista psicológico en los diversos momentos.

El tratamiento psicológico tanto de la familia como de la propia paciente se constituyó a partir de intervenciones psicológicas más o menos extensas (en función de las circunstan- 


\section{Tabla 5. Objetivos específicos e Intervenciones psicológicas con paciente y familiares en los distintos momentos temporales del caso.}

\begin{tabular}{|c|c|c|c|}
\hline & & & If \\
\hline 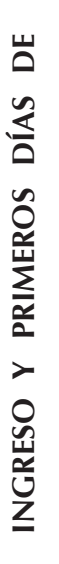 & 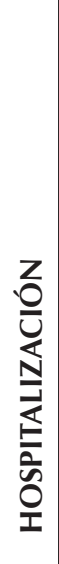 & $\begin{array}{l}\text { Acogida, presentación de psicóloga. } \\
\text { Exploración necesidades de información. } \\
\text { Detección de dificultades en el afronta- } \\
\text { miento de la enfermedad. } \\
\text { Desahogo emocional. Contención. Nor- } \\
\text { malizar y validar. } \\
\text { Fomentar apoyo social (familiar y amigos) } \\
\text { Refuerzo de actividades gratificantes. } \\
\text { Manejo crisis de ansiedad/manejo culpa }\end{array}$ & $\begin{array}{l}\text { Acogida y acceso a servicio de psicología. } \\
\text { Valoración de aspectos familiares que re- } \\
\text { quieren intervención y seguimiento. } \\
\text { Desmontar pacto silencio cuidadosamente } \\
(7) . \\
\text { Fomento de iniciativas que reduzcan ansie- } \\
\text { dad familiar. } \\
\text { Conciliación aspectos familiares (domicilio } \\
\text { y hospitalarios). }\end{array}$ \\
\hline 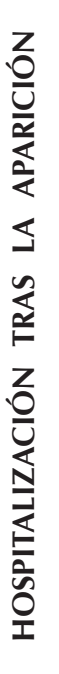 & 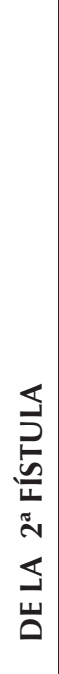 & $\begin{array}{l}\text { Resaltar logros alcanzados. } \\
\text { Fomentar implicación y Locus Control po- } \\
\text { sitivo interno. } \\
\text { Canalización de angustia. Priorización en } \\
\text { preocupaciones. } \\
\text { Narrativa de situaciones. } \\
\text { Manejo de ambivalencia familiar/actua- } \\
\text { ción sobre disonancia cognitiva. } \\
\text { Tolerancia a dormir sola (sin petición fami- } \\
\text { liar de acompañamiento) }\end{array}$ & $\begin{array}{l}\text { Favorecer refuerzo de logros a la paciente. } \\
\text { Comentar objetivos de intervención psico- } \\
\text { lógica y consecución de mismos. } \\
\text { Refuerzo de la capacidad de dar apoyo a } \\
\text { paciente. } \\
\text { Hacer más conscientes de la situación real } \\
\text { y de posible evolución. } \\
\text { Acompañamiento psicológico (ante claudi- } \\
\text { cación familiar) (20). } \\
\text { Pautas de autocuidado y reparto de roles- } \\
\text { horarios. } \\
\text { Pautas manejo de la conducta de la pacien- } \\
\text { te. }\end{array}$ \\
\hline 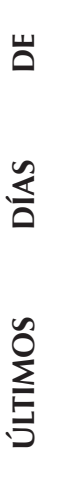 & 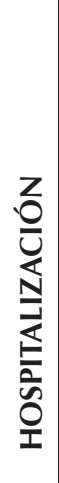 & $\begin{array}{l}\text { Búsqueda proactiva de contacto social. } \\
\text { Fomento de los propios recursos. } \\
\text { Centrarse en aquí-ahora. Limitación de } \\
\text { preocupaciones. } \\
\text { Importancia de pequeñas cosas cotidianas. } \\
\text { Aumento de capacidad de disfrute. } \\
\text { Tolerancia a cambio de planes (salida vo- } \\
\text { luntaria prevista que no se pudo realizar). }\end{array}$ & $\begin{array}{l}\text { Trabajo sobre miedos latentes y explícitos. } \\
(21) \text {. } \\
\text { Intercambio emocional familiar. Canales } \\
\text { de comunicación y explicación de las re- } \\
\text { acciones de otros familiares. } \\
\text { Trabajo sobre aspectos de duelo, previos y } \\
\text { desconcierto ante mejoría temporal. } \\
\text { Dar información sobre discapacidad y de- } \\
\text { pendencia. Emisión de informe psicológico } \\
\text { para la Administración correspondiente. }\end{array}$ \\
\hline
\end{tabular}




\section{Tabla 6. Técnicas psicológicas generales empleadas en el caso.}

\begin{tabular}{|c|c|}
\hline Técnicas con la paciente & Técnicas con la familia \\
\hline Counselling $^{(23)}$ Validación ${ }^{(24)}$ & Counselling $^{(23)} \cdot V$ alidación ${ }^{(24)}$ \\
\hline Animar a expresar miedos y sentimientos ${ }^{(25)}$ & Ventilación y Manejo emocional. \\
\hline Regulación cognitiva y emocional. & de solución de problemas \\
\hline Técnicas de solución de problemas. & decisiones. \\
\hline Psicoeducación. Analogías ${ }^{(26)}$ & Desbloqueo comunicación paciente - familia ${ }^{(25)}$ \\
\hline Reestructuración cognitiva ${ }^{(7)}$ & $\begin{array}{l}\text { Trabajo sobre los conflictos familiares que po- } \\
\text { dían influenciar la situación oncológica fami- }\end{array}$ \\
\hline Control de pensamiento. & 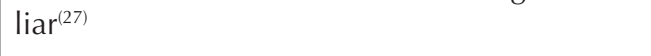 \\
\hline Fomento de recursos de afrontamiento ${ }^{(25)}$ & Psicoeducación. \\
\hline Respiración /relajación ${ }^{(7)}$ & Manejo específico de culpa, ansiedad \\
\hline $\begin{array}{l}\text { Control dolor: distracción cognitiva y física, } \\
\text { soplar, autoinstrucciones externas, derivación } \\
\text { dolor }^{(21)} \\
\text { Higiene de sueño }\end{array}$ & $\begin{array}{l}\text { Trabajo sobre aspectos de pareja (paciente y } \\
\text { esposo) } \\
\text { Proporcionar información útil sobre la enferme- } \\
\text { dad }^{(25)}\end{array}$ \\
\hline
\end{tabular}

cias de cada día). En la tabla 5 se explican de manera pormenorizada en qué consistieron. Se ha seguido el mismo esquema de la tabla anterior concretando en los tres momentos temporales para que se puedan encontrar equivalencias entre ambas tablas. Se ha dedicado la columna de la izquierda a exponer cuál fue la actuación con la paciente y en el lado derecho cuál fue la actuación concurrente con la familia de la misma. Se detallan objetivos específicos y aspectos sobre los que se aplicaron estrategias psicológicas concretas para cada momento.

Las técnicas que se usan en enfermedad oncológica $^{(22)}$ desde el punto de vista psicoterapéutico pueden ser para resolver un problema concreto, como la intervención sobre el dolor, o para mejorar su nivel de adaptación físico y psicológico. Siguiendo progresivamente de lo más general a lo más especifico, se enumeran en la tabla 6, de manera diferenciada las técnicas psicológicas que se emplearon en este caso tanto con la paciente como con la familia y allegados. Se han descrito en bloque porque prácticamente todas las técnicas estuvieron presentes en las tres fases. Se centraron sobre todo en el manejo de emociones y pensamientos tanto en paciente como familia, así como en solución de dificultades, o problemas que fueron surgiendo a lo largo del proceso. Se usó el modelo cognitivo conductual y se aplicaron también los principios del counseIling como herramienta terapéutica específica de la atención a la unidad paciente-familia en Psicooncología.

En este caso como objetivos de intervención psicológica se asumen los propuestos por la Terapia Psicológica Adyuvante ${ }^{(29)}$, adaptados al caso de la paciente:

- Reducir la ansiedad, la depresión y otros síntomas psicológicos,

- Promover en la paciente un sentido de control personal sobre su vida y una participación activa en el tratamiento de la enfermedad.

- Desarrollar estrategias de afrontamiento efectivas para tratar los problemas relacionados con el cáncer (y sus consecuencias)

- Mejorar la comunicación entre el paciente y su pareja y/o otros familiares y alentar la expresión abierta de sentimientos negativos especialmente la ira, canalizándolos. 


\section{Tabla 7. Intervención psicológica familiar postfallecimiento.}

\begin{tabular}{|c|c|c|}
\hline Metodología de intervención psicológica & $\begin{array}{l}\text { Objetivos de intervención } \\
\text { psicológica. }\end{array}$ & $\begin{array}{l}\text { Tareas de seguimiento del } \\
\text { duelo familiar. }\end{array}$ \\
\hline $\begin{array}{l}\text { Entrevistas familiares ( } 3 \text { ocasiones) } \\
\text { con hijas, esposo, nieto, cuidadora } \\
\text { Llamadas telefónicas tras el } \\
\text { fallecimiento (hijas y esposo) } \\
\text { Entrega de dos folletos de duelo: } \\
\text { - Día del deceso: folleto sobre las } \\
\text { reacciones iniciales de duelo } \\
\text { - Después de } 15 \text { días: folleto sobre } \\
\text { las reacciones de duelo posteriores }\end{array}$ & $\begin{array}{l}\text { Valorar estrategias } \\
\text { presentes de duelo. } \\
\text { Prevenir reacciones } \\
\text { desadaptadas. } \\
\text { Favorecer la elaboración } \\
\text { de duelo sano. } \\
\text { Insistir en la comunicación } \\
\text { intrafamiliar }\end{array}$ & $\begin{array}{l}\text { Manejo de la culpa. } \\
\text { Toma de decisiones. } \\
\text { Recuerdo agradecido y } \\
\text { recolocación emocional } \\
\text { del ser querido } \\
\text { Inicio de las } 4 \text { tareas de } \\
\text { duelo }^{(31)}\end{array}$ \\
\hline
\end{tabular}

Cuando la paciente falleció, no se dejó de atender a la familia de manera abrupta. Teniendo en cuenta las características del caso y la confianza que se había establecido con el equipo terapéutico, se consideró necesario el realizar una intervención posterior para favorecer la elaboración del duelo. Esta intervención fue llevada a cabo en la mayor parte por la psicóloga, pero se siguió comunicando al médico responsable del caso. En la tabla 7 se expone de manera esquemática y organizada cuál fue la metodología de la intervención psicológica en este caso, los objetivos psicológicos concretos para esta familia (los deudos) y las tareas psicológicas de seguimiento del duelo familiar.

\section{RESULTADOS OBTENIDOS DE LA INTERVENCIÓN MÉDICO- PSICOLÓGICA}

Una vez expuesto todo el proceso de planificación psicológica y médica, el diseño de intervención psicológica, la metodología empleada, los objetivos generales y específicos, las técnicas psicológicas concretas, vamos a ver qué resultados se produjeron y en qué medida tanto la intervención psicológica por sí sola, como la intervención médico -psicológica fueron eficaces en su aplicación. En este caso y dada la intensa coordinación entre los dos profesionales, podemos decir que los resultados se deben a la actuación de ambos, y a la comunicación diaria entre ambos.

Tanto la familia como la paciente, verbalizaron en varios momentos de la hospitalización su satisfacción hacia la intervención conjugada de ambos (médico y psicóloga). Ambos profesionales también pueden constatar cambios objetivos y positivos en el caso estudiado, que se exponen en la tabla a continuación. Al mismo tiempo otros profesionales que participaron de manera más o menos activa, en el caso transmitieron al médico y/o la psicóloga los aspectos en que percibieron cambios positivos, favoreciendo el manejo de la paciente $y / 0$ familia desde otras vertientes profesionales.

Puede verse en la tabla 8 cómo la paciente puso en marcha recursos psicológicos que le permitieron una mejor estancia hospitalaria, valorar aspectos presentes en su situación (como la presencia de apoyo social casi permanente) y cómo se produjeron mejorías en sueño, alimentación, peticiones adecuadas y no influenciadas por manifestaciones ansiosas. En la familia los cambios fueron en la dirección siguiente: inclusión del esposo de manera más participativa en los cuidados, mejor comunicación en el seno familiar en varias 


\section{Tabla 8. Resultados de la intervención Médico-Psicológica en el caso.}

\begin{tabular}{|c|c|}
\hline $\begin{array}{l}\text { RESULTADOS EN PACIEN } \\
\text { CONJUNTA }\end{array}$ & VTA \\
\hline $\begin{array}{l}\text { * Mejor tolerancia a la soledad y frustración. } \\
\text { Mejor capacidad para posponer refuerzo y } \\
\text { mejor manejo de pensamientos negativos. } \\
\text { * Mayor valoración del acompañamiento } \\
\text { familiar. } \\
\text { * Control de ansiedad y estabilización del } \\
\text { estado anímico. } \\
\text { * Mejor adaptación al proceso oncológico. } \\
\text { * Mejores recursos afrontamiento. } \\
\text { * Mejoría en apetito y sueño } \\
\text { - Menor preocupación por no tener } \\
\text { Buen etiquetaje emocional y mayor } \\
\text { capadad para hablar de emociones. } \\
\text { manteamiento de temas espirituales de } \\
\text { manera adecuada y ordenada. }\end{array}$ & 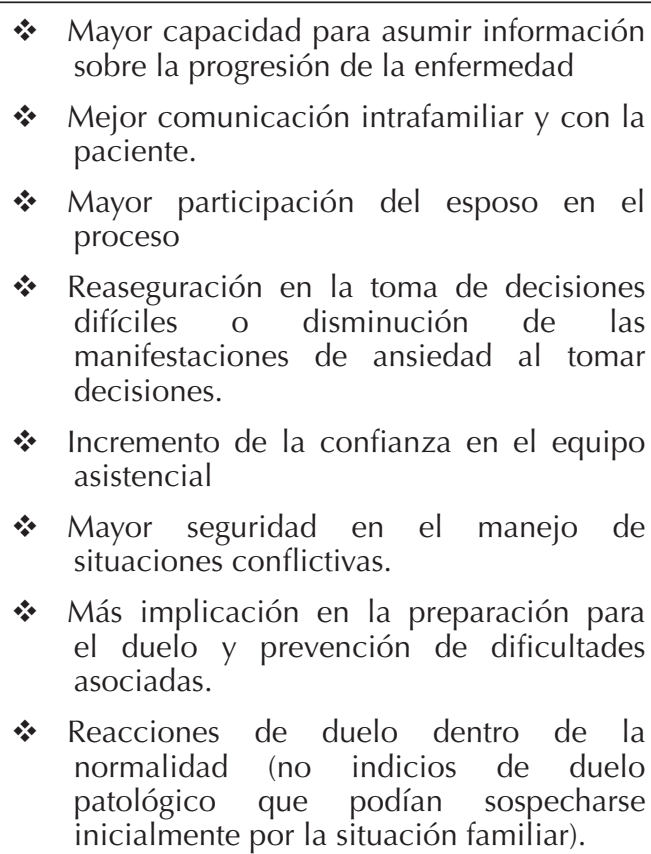 \\
\hline
\end{tabular}

direcciones y comunicación con el equipo terapéutico.

El counselling empleado en la intervención con esta paciente (y su familia), promueve la motivación, el cambio desde la elección, el respeto a sí mismos y a los demás ${ }^{(19)}$.

La familia necesita apoyo y comunicación para poder apoyar al paciente y clarificar ideas acerca de las opciones terapéuticas; por ello es fundamental (para implicarles en la dinámica de la enfermedad) las reuniones periódicas entre médico y familia ${ }^{(32)}$ que en este caso se realizaron en varias ocasiones con el acompañamiento de la psicóloga.

Se trabajó con la familia a lo largo de todo el proceso; como la familia no tuvo posibilidad de despedirse en el momento en que se produjo la muerte, la profesional de psicología enfocó la intervención hacia la reparación de los sentimientos negación asociados ${ }^{(33)}$.

\section{DISCUSIÓN DEL CASO}

En este caso se pudo realizar una intervención conjunta, coordinada, consensuada, eficaz y eficiente, desde el mismo momento del ingreso hasta el fallecimiento de la paciente en el centro, así como en los seguimientos a los familiares tras el óbito.

Se partió del modelo de Buckman sobre Comunicación de malas noticias ${ }^{(38)}$, pero haciéndolo extensivo a la familia, es decir con ambos se siguieron los pasos de preparar el planteamiento, saber qué saben (paciente y familia), qué quieren saber (ambos), aportar la información (requerida por paciente y/o familia en cada caso), asumir las reacciones (de ambos) y planificar el futuro (de distinta manera en ambos roles).

En cuanto al control de síntomas, la familia apreció peor control de síntomas que el perso- 
nal sanitario; esto coincide con lo que corroboraron experimentalmente algunos autores ${ }^{(35)}$; sin embargo valoraron positivamente la atención prestada a la paciente.

Se aprecia como paradoja que cuando la paciente verbalizaba mayor bienestar psicológico era cuando se encontraba mal físicamente; y se apreciaba estabilidad psicológica cuando empeoraban los aspectos físicos. Se pudieron abordar las preocupaciones de la paciente y familia una vez jerarquizadas; se adaptó la propuesta de Barreto et al. ${ }^{(23)}$ para una sesión de counselling (para las intervenciones con paciente y/o familia).

Siempre se respetó la autonomía de la paciente y su capacidad de decisión ${ }^{(6)}$. En este sentido se trabajó inicialmente con la familia en relación al pacto de silencio, que es algo que proviene del miedo al diálogo con la paciente, no saber qué decirle, perder el control emocional...(7)

Se trabajó miedo a la muerte en los días previos al fallecimiento, en relación a los temas preocupantes para la paciente sobre su proceso oncológico, situación de los familiares supervivientes $^{(36)}$, pero no se pudo completar el trabajo en las condiciones previstas porque sobrevino la muerte de manera inesperada, cuando se trabajaba para el descanso nocturno sin compañía de familiares.

La paciente tenía más recursos psicológicos de los que mostró inicialmente. A lo largo de la intervención médico psicológica se objetivó un buen uso del sentido del humor progresivamente.

Por otro lado y como plantean otros autores $^{(35)}$, en cuidados paliativos el plantear obtener medidas psicométricas de variables de paciente y/o familia se convierte en una dificultad por las condiciones psicofísicas del paciente y en ocasiones, de la familia.

Se realizó intervención familiar en duelo, teniendo en cuenta las circunstancias de la enfermedad de la paciente y de la familia en concreto $^{(37)}$.

Partimos de un modelo de trabajo en equipo donde debe existir comunicación fluida y continuada $^{(11)}$ que permita la reformulación de objetivos con la paciente y/o familia y la adaptación a la realidad del caso, especialmente cuando se trata de enfermedad avanzada.

En cuando a la figura del psicólogo en Cuidados Paliativos, se ha avanzado pero todavía queda mucho por hacer. Algunos autores ${ }^{(38)}$ planteaban en 2001 la importancia de demandar la intervención del psicólogo de manera precoz como medio de prevención antes de que aparezcan estados emocionales intratables; podemos decir que en este caso la incorporación de los aspectos psicológicos se hizo con este objetivo esencial de terapéutica psicológica preventiva. Desde otros autores ${ }^{(39)}$ se aboga por la figura del psicólogo como investigador, asesor, educador y como un clínico que contribuye al establecimiento del plan de cuidados y en la toma de decisiones del equipo asistencial, identificando vías de sufrimiento y vías para paliarlo.

Se considera la propuesta teórica de algunos autores ${ }^{(29)}$ con la Terapia Psicológica Adyuvante (TPA) en pacientes oncológicos, que se basa en la terapia cognitiva de Beck pero más breve y enfocada al problema de tipo emocional, interpersonal (como relación de pareja), de imagen corporal, fomentando el componente psicoeducativo ${ }^{(22)}$.

Consideramos que para que la coordinación entre médico y psicóloga se pueda hacer con las debidas garantías es necesario que se reúnan varias condiciones:

- Actitud de ambos perfiles por el trabajo en equipo y la interdisciplinariedad.

- Métodos de trabajo con características similares: orientación al paciente y a la familia, respeto, comunicación fluida.

- Por parte de psicóloga: conocimientos de la terminología médica, y del funcionamiento y organización hospitalarios, para favorecer la comunicación y el intercambio de estrategias de intervención, no intromisión en tareas médicas ni de enfermería, con refuerzo de las mismas ante paciente y/o familia.

- Por parte de médico tratante: conocimientos de la función de psicología en una unidad de cuidados paliativos, habilidades sociales y 
de comunicación (empatía, escucha activa, uso de silencios funcionales, empleo adecuado de Comunicación no verbal) en las actuaciones con paciente y familia.

- Trabajo conjunto desde la complementación de actuaciones de diversos perfiles profesionales, desde una perspectiva integral del tratamiento del paciente.

- Comunicación y transmisión continúa de información entre ambos para colaboración conjunta; teniendo en cuenta siempre que el médico es el responsable del caso.

- Confianza del médico hacia las intervenciones de la psicóloga, cuando ésta realizaba intervenciones en un horario distinto. La psicóloga puede reforzar ante la paciente información médica transmitida a la paciente por el facultativo en presencia de psicóloga. También puede hacer de enlace con enfermería para transmitirles indicaciones médicas y autorizaciones verbalizadas por el médico en presencia de personal del hospital, pero no consignadas por escrito.

- Sinergia en las actuaciones de ambos. Acuerdo tácito y explicito en el manejo de ciertas situaciones.

Consideramos que todas estas condiciones se han dado en el caso descrito en este artículo y han favorecido el buen desarrollo del mismo.

Para que se obtuvieran resultados positivos en paciente y familia, es necesario resaltar los factores que contribuyeron a los mismos, desde diversos elementos:

- Aspectos favorecedores (por parte de la paciente). Mecanismos adaptación: negación y disociación.

- Aspectos favorecedores (por parte de la familia y allegados): buen apoyo percibido por parte de la paciente (afectivo e instrumental), cuidadora implicada y cariñosa con la paciente; poder salir de la habitación y pasear por el hospital, ponerle ropa propia (personalización de su rutina cotidiana).

- Aspectos favorecedores (en relación al hospital): percepción positiva de intervención médico-psicológica en conjunto; Instalaciones nuevas/habitación individual, libertad para ele- gir comida o complementar dieta con alimentos aportados por la familia.

\section{CONCLUSIONES}

Tras la exposición del caso en toda su longitud, proponemos las siguientes conclusiones:

- Se realizó un trabajo fundamental con paciente y familia (como unidad de intervención), para conseguir los objetivos terapéuticos previstos.

- La coordinación médico-psicóloga consiguió resultados positivos en la adaptación de la paciente y la familia a la progresión de la enfermedad.

- La figura del psicólogo es valorada dentro de la organización hospitalaria. Abogamos por la promoción de la figura del psicólogo dentro del ámbito de Psicología de la Salud y de la Psicooncología, de manera concreta en los Cuidados Paliativos, por la importancia de su función. No sólo como apoyo al paciente ingresado, sino también a la familia y allegados, y también al equipo asistencial (médicos, enfermeras, auxiliares y otros profesionales que se encuentran en contacto directo con el sufrimiento familiar).

- Se destaca la importancia de la atención psicológica desde el momento del ingreso. Asociado a la posibilidad de acompañar a lo largo de todo el proceso.

- Se llevó a cabo un buen trabajo en equipo interdisciplinar y una comunicación fluida de manera diaria, ajustándose a las necesidades que iban apareciendo en la unidad objeto de intervención.

- Se realizó fluidamente la coordinación con otros profesionales (intra y extra hospitalarios).

\section{REFERENCIAS BIBLIOGRÁFICAS}

1. Eltabbakh GH, Piver MS. Extraovarian primary peritoneal carcinoma. Oncology (Willinston Park) 1998; 12: 813-25.

2. De Vita H, Rosenberg S. Cancer Principles and Practice. Oncology. Vol. 2. 8th. Wolters Klower Health. Lippincott Williams \& Wilkins. 
3. Bloss JD, Liao SY, Buller RE, Manett A, Berman ML, McMeekin S, et al. Extraovarian peritoneal serous papillary carcinoma: A case-control retrospective comparison to papillary adenocarcinoma of the ovary. Gynecol Oncol 1993; 50: 347-51. Doi:10.1006/gyno.1993.1223 Obstet Gynecol

4. Feuer GA, Shevchuk M, Calanog A. Normal-sized ovary carcinoma syndrome. Obstet Gynecol 1989; 73: 786-92.

5. Swerdlow M. Mesothelioma of the pelvic peritoneum resembling papillary cystadenocarcinoma of the ovary; case report. Am J Obstet Gynecol 1959;77:197-200

6. Cambra Lasaosa FJ, Masia Clavel J, De Castro Martínez J, Etayo Arrondo J, Galán González-Serna JM, Núñez y Pérez JE et al. Humanizar el proceso de morir. Sobre la ética de la asistencia en el morir. Orden Hospitalaria de San Juan de Dios. Comisión Interprovincial. Fundación Juan Ciudad. 2007.

7. Schröder M. Impacto emocional y tratamiento psicológico durante la fase terminal. En Die Trill, M, editora. . Psicooncologia. Madrid: Ades Ediciones, 2003. p. 629-45.

8. Arranz E, Arango, MO, Acinas MP, Pérez AM, Antón S. Estudio descriptivo retrospectivo sobre intervención medico-psicológica con familiares de pacientes en cuidados paliativos oncológicos. Hospital San Juan de Dios. Burgos. Comunicación Póster IV Jornadas de Cuidados Paliativos de Castilla y León. PACYL. Salamanca 29 y 30 de octubre 2010.

9. Sanz J, Modolell E. Oncología y Psicología: un modelo de interacción. Psicooncología 2004; 1 (1): 3-12.

10. Acinas MP. Protocolos de actuación de psicología del Hospital San Juan de Dios. 2009. Burgos: Hospital Hermanos San Juan de Dios.

11. Ibañez C, Ávila R, Gómez MD, Díaz R. El equipo interdisciplinar como instrumento básico en cuidados paliativos: a propósito de un caso. Psicooncología, 2008; 5 (2-3): 439-457.

12. Antón Torres A, Calderero Aragón V, Puértolas Hernández, T. Influencia del control del dolor y otros síntomas en el duelo. En Camps Herrero C, Sánchez Hernández PT, editores. Duelo en Oncología. Madrid: SEOM, 2007: 49-62.

13. Karnofsky DA, Burchenal JH. The clinical evaluation of chemotherapeutic agents in cancer. In: MacLeod CM, editor. Evaluation of chemotherapeutic agents, New York, Columbia University Press, 1949; pp. 191-205.

14. Oken MM., Creech, RH., Tormey DC, Horton J, Davis TE, McFadden ET, et al. Toxicity And Response Criteria Of The Eastern Cooperative Oncology Group. Am J Clin Oncol 1982; 5:649-55. Doi:10.1097/00000421198212000-00014

15. Van Swieten, KC. Koudsta al PJ, Visser MC, Schouten HJA, Van Gij J. Interobserver agreement for the assessment of handicap in stroke patients. Stroke 1988; 19 (5):60417. Doi:10.1161/01.STR.19.5.604

16. Baños RM, Perpiñá C. Exploración Psicopatológica. Guías técnicas de Psicología Clínica. Síntesis: Madrid, 2004

17. Carrulla Torrent J, Pérez Lianes JP, Guzmán Pérez, M. Despedida del paciente en la fase final de la vida. En: Camps Herrero C, Sánchez Hernández PT, editores. Duelo en Oncología. Madrid: SEOM, 2007: 35-48.

18. American Psychiatric Association. DSMIV- TR. Manual Diagnóstico y Estadístico de las Enfermedades Mentales IV. Texto Revisado. Barcelona: Masson. 2004.

19. Pascual López A, García Campayo, J. La familia y la enfermedad crónica. En: García Campayo J, editor. La familia y el médico de familia. Elementos básicos de intervención desde atención primaria. Madrid-Barcelona: Ediciones Mayo, 2005. p. 144-51.

20. Rocamora A. Un enfermo en la familia. Claves para la intervención psicológica. Madrid: San Pablo, 2000. 
21. 21- Florez Lozano JA. Aspectos psicoafectivos del enfermo terminal: atención ética integral: Comunicación en Ciencias de la Salud. Barcelona: División de Biomedical Systems Group, 2001.

22. Romero Retes R. Intervención Psicológica en el paciente oncológico crónico. En Monsalve Dolz V, Soriano Pastor J, Carbajo Álvarez E, Santolaya Ochando F, editores. Guía práctica de la psicología de la salud en el ámbito hospitalario. Colegio Oficial de Psicólogos de Valencia. [En línea] 2009. [fecha de acceso 11.02.2010] URL disponible en: http://www.cop-cv.org/db/ docu/37751996.pdf

23. Barreto Martin MP, Saavedra Muñoz G, Díaz Cordobés JL, Vázquez N, Yi P. Intervención psicológica en el final de la vida. FOCAD. 2009. Plataforma Consejo General de Colegios Oficiales de de Psicólogos.

24. Arranz P, Barbero J, Barreto P, Bayés, R. Intervención emocional en Cuidados Paliativos. $2^{\text {a }}$ ed. Barcelona: Ariel. 2003

25. Comas MD, Schröder M, Villaba O. Intervención psicológica en una unidad de cuidados paliativos. En: Remor R. Arranz P; Ulla, S, editores. El psicólogo en el ámbito hospitalario. Bilbao: Desclée de Brouwer; 2003. p. 777-813.

26. Rojí B, Cabestrero R. Entrevista y sugestiones indirectas: entrenamiento comunicativo para jóvenes psicoterapeutas. Cuadernos de la UNED. UNED ediciones: Madrid. 2005.

27. Die Trill M. Intervención Psicológica en un servicio de Oncología. En: Remor R, Arranz P, Ulla S, editores. El psicólogo en el ámbito hospitalario. Bilbao: Desclée de Brouwer; 2003. p. 622-40.

28. Navarro Góngora J. Enfermedad y familia. Manual de intervención psicosocial. Barcelona: Paidós, 2004.

29. Greer S, Moorey S. Adjuvant Psychological Therapy for cancer pacients. Palliat Med 1997; 11 (3): 240-4. Doi:10.1177/026921639701100309

30. Cabodevilla Eraso I. Las reacciones de duelo. En: Die Trill, M. Psicooncologia. Madrid: Ades Ediciones, 2003: 647-57.

31. Worden JW. Tratamiento del duelo: asesoramiento psicológico y terapia. Barcelona. Paidós, 1997.

32. Camps Herrero C, Caballero Díaz C, Blasco Cordellat C. Comunicación y duelo. Formas del duelo. En: Camps Herrero C, Sánchez Hernández PT, editores. Duelo en Oncología. Madrid: SEOM, 2007. p.17-33.

33. García Campayo J, Alda M. Procesos de duelo en las familias. En: García Campayo J, editor. La familia y el médico de familia. Elementos básicos de intervención desde Atención Primaria. Madrid-Barcelona: Ediciones Mayo, 2005: 107-16.

34. Buckman R. How to break bad news. A guide for health care professionals. Baltimore: John Hopkins, 1992.

35. Maté J, Bayés R, González Borboteo J, Muñoz S, Moreno F, Gómez- Batiste, X. ¿A qué se atribuye que los enfermos oncológicos de una unidad de cuidados paliativos mueran en paz? Psicooncología, 2008; 5 (2-3): 303-21.

36. Pascual López A, García Campayo, J. La familia ante la enfermedad terminal. En García Campayo J, editores. La familia y el médico de familia. Elementos básicos de intervención desde atención primaria. Madrid-Barcelona: Ediciones Mayo, 2005: 152-8.

37. Virizuela Echaburu JA, Aires Gonzalez MM, Duque Amusco A. Intervención familiar en duelo. En: Camps Herrero C, Sanchez Hernandez PT., editores. Duelo en Oncologia. Madrid: SEOM, 2007. p. 155-68.

38. Romero C, Álvarez M, Bayés R, Schröder M. ¿Cuándo se pide la intervención del psicólogo en una unidad de Cuidados Paliativos? Med Pal 2001; 8 (4): 170-2.

39. Mateos J. Trabajo interdisciplinar con los pacientes al final de la vida. En: Remor $R$, Arranz P, Ulla S, editores. El psicólogo en el ámbito hospitalario. Bilbao: Desclée de Brouwer; 2003: 815-30. 
